\title{
LES ÉLECTIONS EUROPÉENNES DE JUIN 2009 EN ESPAGNE : PREMIÈRE APPARITION D'UN VOTE SANCTION
}

\author{
Joan Marcet \\ De Boeck Supérieur | Revue internationale de politique comparée
}

2009/4 - Vol. 16

pages 685 à 696

ISSN 1370-0731

Article disponible en ligne à l'adresse:

http://www.cairn.info/revue-internationale-de-politique-comparee-2009-4-page-685.htm

Pour citer cet article :

Marcet Joan, « Les élections européennes de juin 2009 en Espagne : première apparition d'un vote sanction », Revue internationale de politique comparée, 2009/4 Vol. 16, p. 685-696. DOI : 10.3917/ripc.164.0685

Distribution électronique Cairn.info pour De Boeck Supérieur.

(c) De Boeck Supérieur. Tous droits réservés pour tous pays.

La reproduction ou représentation de cet article, notamment par photocopie, n'est autorisée que dans les limites des conditions générales d'utilisation du site ou, le cas échéant, des conditions générales de la licence souscrite par votre établissement. Toute autre reproduction ou représentation, en tout ou partie, sous quelque forme et de quelque manière que ce soit, est interdite sauf accord préalable et écrit de l'éditeur, en dehors des cas prévus par la législation en vigueur en France. II est précisé que son stockage dans une base de données est également interdit. 


\title{
LES ÉLECTIONS EUROPÉENNES DE JUIN 2009 EN ESPAGNE: \\ PREMIÈRE APPARITION D'UN VOTE SANCTION
}

\author{
Joan MARCET
}

Le contexte des élections européennes de juin 2009 en Espagne a été marqué par l'installation progressive et profonde de la crise économique et une crise politique ouverte dès le début du second mandat du gouvernement socialiste de J. L. Rodríguez Zapatero en 2008. La campagne électorale de juin 2009 fut très polarisée sur l'affrontement entre les deux grands partis : PSOE et PP. Les résultats montrent un léger recul socialiste par rapport aux élections européennes de 2004, plus important dans les zones urbaines, profitant inégalement au PP selon les Communautés. Enfin pour la première fois s'est exprimé de façon claire dans des élections européennes un «vote sanction » contre le gouvernement, peu utilisé en Espagne jusqu'à présent.

Les élections européennes se sont déroulées en Espagne un peu plus d'un an après les élections législatives de mars 2008, qui avaient confirmé la victoire électorale du Partido Socialista Obrero Español (PSOE) et la personne de son leader - José Rodríguez Zapatero - pour un second mandat à la tête du gouvernement espagnol. À ce moment, le vote socialiste atteignait $43,8 \%$ des suffrages exprimés et dominait d'un peu plus d'un million de voix le score du Partido Popular ( $P P$ ) qui n'arrivait qu'en seconde position avec 39,9\%. Malgré cette avance de près de quatre points du PSOE, le gouvernement de Rodríguez Zapatero s'est retrouvé très vite sans majorité parlementaire stable, devant faire face à une crise économique dont les premiers indices étaient passés inaperçus durant la campagne législative de 2008 mais qui allait se manifester avec une envergure exceptionnelle au cours des mois suivants.

Ainsi le contexte dans lequel ont eu lieu les élections européennes de juin 2009 a été marqué par l'installation progressive et profonde de la crise économique, à laquelle s'est ajoutée une crise politique ouverte dès le début du second mandat de J. L. Rodríguez Zapatero. 


\section{Un contexte de profonde crise économique et de crise politique}

La crise financière puis économique a en effet frappé particulièrement durement l'Espagne. La chute du Produit Intérieur Brut atteignit en un an plus de cinq points, passant de $+2,5$ points en mars/avril 2008 à -3 points en mai 2009. Cette chute du PIB s'est accompagnée d'un fort accroissement du chômage et d'une évolution négative de la consommation et des prix.

Le chômage a atteint, pendant le premier trimestre 2009, les quatre millions de personnes selon l'Enquête de population active ce qui représente une hausse de presque huit points par rapport au premier trimestre 2008. Avec un taux de chômage de $18 \%$ en avril 2009, l'Espagne se situe en tête des pays de l'Union européenne, avec une proportion de chômeurs dans sa population active pratiquement équivalente au double de la moyenne de l'Union.

Le recul de l'emploi s'accompagne d'une chute de la consommation et d'une évolution négative des prix, qui, en mai 2009, placent l'Espagne dans une situation de déflation, après trois mois de taux négatifs consécutifs. Cette évolution négative des prix est encore plus remarquable si on compare la situation de l'Espagne avec celle du reste de la zone euro, qui partant d'une inflation plus faible ne connaît pas de taux négatifs des prix en mai $2009^{1}$.

Dans un contexte aussi grave de crise on ne s'étonnera pas que l'indicateur du Centro de Investigationes Sociológicas ${ }^{2}$ mesurant l'optimisme des citoyens espagnols vis-à-vis de l'évolution de l'économie ait chuté de presque 20 points, passant de $42,2 \%$ au premier trimestre 2008 à $25,4 \%$ au premier trimestre 2009. Rappelons que ce même indicateur avait frôlé les $60 \%$ au début de l'actuelle décennie !

À l'ampleur prise par la crise économique espagnole - sujet de nombreux débats politiques et sociaux très médiatisés tout au long des douze mois précédant les élections européennes - se sont ajoutés de nombreux éléments de crise politique qui ont aussi marqué le contexte de ces élections. Leurs effets ont concerné tant le parti socialiste que le principal parti d'opposition, le Partido Popular.

En mars 2008, le Partido Popular avait été battu pour la deuxième fois consécutive lors d'élections législatives. Cette seconde défaite avait placé son leader et candidat à la tête du gouvernement - Mariano Rajoy - face à une crise de légitimité en tant que leader du principal parti d'opposition qu'il

1. Voir les données de l'Instituto Nacional de Estadística (INE) qui publie des tableaux comparés de l'Espagne avec l'union monétaire.

2. Voir les indicateurs du Barómetro de opinión sur le site Internet www.cis.es. 
ne résolut que formellement lors du Congrès du parti en juin 2008. Son sort final et son maintien à la tête du $P P$ demeuraient liés à un rétablissement électoral à venir. Les élections des Autonomies de Galice et du Pays Basque, qui se tinrent en avril 2009 à peine deux mois avant les élections européennes lui offrirent un premier répit puisque le $P P$ réussit à conquérir à nouveau le gouvernement de Galice et contribua de façon décisive à la formation du premier gouvernement basque qui ne fut pas présidé par le Partido Nacionalista Vasco $(P N V)$ en favorisant l'investiture du candidat socialiste ${ }^{3}$ Les élections européennes de juin apparaissaient donc comme cruciales pour la consolidation ou non du leadership de M. Rajoy au sein du PP.

De son côté, le gouvernement socialiste, qui ne disposait que d'une faible et instable majorité parlementaire, n'avait cessé de voir sa crédibilité diminuer tout au long des mois précédents les élections européennes. Les doutes face aux mesures prises pour faire face à la crise économique - qui entraînèrent le remplacement du ministre de l'économie et des finances et de son cabinet - ainsi que diverses erreurs et maladresses politiques provoquèrent une forte perte de crédibilité du Gouvernement et de son Président, le leader socialiste J. L. Rodríguez Zapatero (Tableau 1).

Tableau 1 : L'évolution des jugements concernant le gouvernement, son président, le PP et son leader : avril 2008 - avril 2009

\begin{tabular}{|c|c|c|c|}
\hline & Avril 2008 & Avril 2009 & $\begin{array}{l}\text { Évolution } \\
2008-2009\end{array}$ \\
\hline $\begin{array}{l}\text { Le Gouvernement est évalué... } \\
\text { - Bien et très bien } \\
\text { - Mal et très mal }\end{array}$ & $\begin{array}{l}34,7 \% \\
20,9 \%\end{array}$ & $\begin{array}{l}15,9 \% \\
39,2 \%\end{array}$ & $\begin{array}{l}-18,8 \\
+18,3\end{array}$ \\
\hline $\begin{array}{l}\text { Note moyenne d'évaluation } \\
\text { du Président du gouvernement } \\
\text { J. L. R. Zapatero }\end{array}$ & 5,58 & 4,43 & $-1,15$ \\
\hline $\begin{array}{l}\text { L'Opposition (PP) est évaluée... } \\
\text { - Bien et très bien } \\
\text { - Mal et très mal }\end{array}$ & $\begin{array}{l}14,4 \% \\
41,3 \%\end{array}$ & $\begin{array}{c}9,5 \% \\
51,6 \%\end{array}$ & $\begin{array}{c}-4,9 \\
+10,3\end{array}$ \\
\hline $\begin{array}{l}\text { Note moyenne d'évaluation du } \\
\text { leader du PP, M. Rajoy }\end{array}$ & 4,24 & 3,54 & $-0,70$ \\
\hline
\end{tabular}

Source : L'auteur à partir des enquêtes (Barómetros) du Centro de Investigaciones Sociológicas n²761 (avril 2008) et $n^{\circ} 2798$ (avril 2009)

3. La stratégie électorale et politique du $P P$ était de porter à la tête du gouvernement basque le candidat gagnant d'un des deux partis non nationalistes : $P S E-P S O E$ ou $P P$ selon les résultats. Dans l'hypothèse où le PNV serait arrivé en tête des élections, il n'aurait pas eu de majorité parlementaire possible. 
La perte de confiance dans le gouvernement et la chute de la note moyenne attribuée au leader socialiste n'ont pas entraîné la montée de la confiance dans le Partido Popular pas plus que celle dans son leader. L'un comme l'autre connaissent un recul des jugements positifs à leur égard. L'absence d'une proposition suffisamment claire et visible d'alternative économique à la politique de crise du gouvernement de J. L. R. Zapatero a certainement privé le $P P$ du transfert de confiance de l'opinion espagnole en sa faveur. Ceci malgré le positionnement politique plus solide et plus recentré qu'il a offert après son Congrès du juin 2008.

Les indicateurs de satisfaction de la situation politique des Barometros' du CIS n'ont pas arrêté de chuter pendant la dernière année. Après une courte et modérée reprise de la satisfaction coïncidant avec les élections législatives de mars 2008 (47,5\%), la perception positive des citoyens de la situation politique de l'Espagne s'est de nouveau dégradée pour s'établir à $35,2 \%$ au moment des élections européennes. Crise économique et éloignement du gouvernement des citoyens ont certainement marqué le déroulement des élections européennes.

\section{Une campagne électorale dominée par des enjeux internes}

Dans un tel contexte politique, on ne peut s'étonner que la campagne électorale pour les élections européennes se soit principalement développée sur des thèmes domestiques. Cette tendance s'est vue favorisée par l'existence d'une circonscription de vote unique qui permit la confrontation directe des leaders des grands partis nationaux - PSOE et $P P$ - au détriment du choix des candidats au Parlement européen.

La profondeur de la crise économique, ainsi que les jugements portés sur l'action du gouvernement de Rodriguez Zapatero, fournir les principaux thèmes de controverse électorale de la campagne. S'y ajouta la possibilité pour les forces politiques nationalistes et de rayonnement sub-étatique d'introduire des thèmes à dimension régionale ${ }^{4}$ visant à préparer leurs futures campagnes pour les élections autonomiques de 2010 en Catalogne ou bien encore visant à renouer avec leurs succès d'avril 2009 aux élections autonomiques basques et galiciennes.

La campagne électorale fut très polarisée sur l'affrontement entre les deux grands partis nationaux. Le $P P$ utilisa tous ses arguments de critique

4. Nous nous référons concrètement à la Coalición por Europa, composée principalement par Convergència i Unió et le Partido Nacionalista Vasco, et à la Coalicion Europea de los Pueblos-Verdes, composées principalement par Esquerra Republicana de Catalunya et le Bloque Nacionalista Galego. Voir «Les partis autonomistes : vers la disparition de l'avantage des élections européennes » par Lieven De Winter et Margarita Gómez-Reino. 
de la gestion de la crise par le gouvernement, au détriment d'une offre programmatique spécifique et crédible de lutte contre la crise. Le Parti socialiste et le gouvernement défendirent leur gestion de la crise, annonçant de nouvelles propositions de modernisation sociale, parmi lesquelles la réforme de la législation sur l'avortement fut la plus frappante 5 . Parallèlement les socialistes développèrent à nouveau l'argument de la peur du retour de la droite symbolisée par le $P P$, argument qui avait fonctionné à leur avantage pour les élections législatives de 2008.

Les autres partis nationaux, Izquierda Unida (IU) et Union Progreso y Democracia $(U P y D)^{6}$ et les partis nationalistes ${ }^{7}$ orientèrent leurs combats et leurs campagnes dans l'espoir d'apparaître sur la scène électorale très monopolisée par les deux grands partis.

Les grandes questions et les aspects clés de l'orientation politique européenne n'apparurent que marginalement durant cette campagne bien que tous les partis signalèrent la grande importance de ces élections pour le projet européen et le rôle déterminant du parlement européen qui devait être élu. Les appels à l'Europe sociale du PSOE, ceux aux racines et valeurs chrétiennes de l'Europe du $P P$, la défense du local dans le cadre européen par le $C i U$ et le $P N V$ ou encore la défense des valeurs constitutives de la gauche et des verts de Izquierda Unida et de Iniciativa per Catalunya Verds furent relégués au second plan d'une confrontation médiatique très centrée sur la politique intérieure espagnole. Selon quelques enquêtes par sondage postélectorales, ils n'influencèrent que $17,6 \%$ des citoyens qui participèrent au scrutin ${ }^{8}$.

\section{Les résultats de l'élection}

\section{La participation}

Le taux de participation au scrutin atteint $44,9 \%$, soit un niveau légèrement au-dessus de la moyenne des pays membres de l'Union et proche de celui enregistré lors des élections européennes de $2004(45,1 \%)^{9}$. Toutefois le déficit de participation est net par rapport aux élections législatives qui mobilisent historiquement entre 70 et $75 \%$ des électeurs inscrits, avec des

5. La mesure phare du projet de réforme était l'introduction de l'avortement sans consentement parental entre 16 et 18 ans.

6. Unión Progreso y Democracia $(U P y D)$ est un parti nationaliste mais pas anti-européen. Il s'est présenté pour la première fois aux élections de mars 2008 , obtenant un siège qu'il a réussit à maintenir lors de ces élections européennes.

7. Voir note 4.

8. Voir l'étude postélectorale (Publiscópio) du quotidien El Publico du 16 juin 2009.

9. Voir « La participation électorale : un déficit inégalé » par Anne Muxel. 
pointes qui ont pu atteindre $80 \%$ (aux élections du 1982). C'est également un niveau de participation très inférieur à ceux enregistrés lors des premières élections européennes. Il faut souligner cependant que, tant en 1987 qu'en 1994 et 1999, celles ci avaient coïncidé avec d'autres scrutins municipaux ou régionaux plus mobilisateurs (Graphique 1).

Graphique 1 : Comparaison de la participation électorale aux élections européennes et législatives : 1987-2009

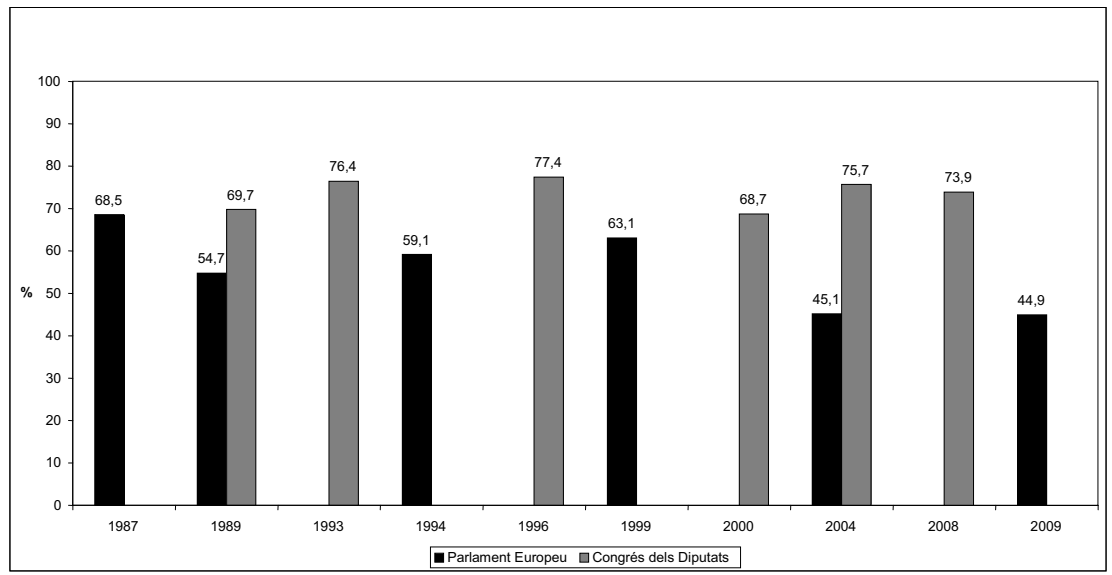

Source : ICPS à partir de données du Ministère de l'Intérieur

Le résultat finalement obtenu de $44,9 \%$ de participation se situa nettement au dessus des prévisions annoncées par les divers sondages et enquêtes préélectorales ${ }^{10}$. Ce dépassement des estimations peut être attribué autant à un accroissement de la conviction de l'importance sui generi des élections pour le Parlement européen qu'à la mobilisation produite par la confrontation entre les deux principaux partis. Les données des études postélectorales ${ }^{11}$ nous indiquent que l'abstention a été sensiblement plus forte chez les jeunes que chez les plus âgés. Les tranches d'âge entre 18-25 ans et 30-44 ans déclarent s'être abstenues respectivement à $45 \%$ et à $44 \%$ tandis que les tranches d'âge entre 45-59 ans et de 60 ans et plus déclarent s'être abstenues à $38 \%$ et $22 \%$. En revanche, selon cette étude, il y aurait eu une répartition quasi parfaite des abstentionnistes entre les différents partis politiques.

La même enquête révèle que les électeurs du $P P$ furent nettement plus motivés par le souhait de contrer la victoire du PSOE (58\%) et de soutenir leur leader M. Rajoy (55\%) que par celui de défendre les idées du $P P$ sur

10. Voir par exemple l'Eurobaromètre $\mathrm{n}^{\circ} 71$ (janvier-févier 2009) qui prévoyait une participation en Espagne de $27 \%$. D'autres sondages la situaient entre 30 et $35 \%$.

11. Voir l'Étude postélectorale (Publiscopio) du périodique El Público du 16 juin 2009. 
l'Europe (51\%). À l'inverse les électeurs du PSOE furent nettement plus déterminés par leur désir d'appuyer le Président du gouvernement (66\%) et les idées de leur parti sur l'Europe (57\%) que par le souhait d'éviter la victoire de l'adversaire $P P(48 \%)$. Ces motivations de vote n'eurent aucune influence sur le vote de $46 \%$ des électeurs socialistes et $45 \%$ des électeurs du PP.

Bien que toutes les enquêtes soulignent l'attitude très positive des citoyens espagnols vis-à-vis de l'Europe ${ }^{12}$, ce sont en 2009 les thèmes et facteurs relevant de la scène politique espagnole qui influencèrent le plus les électeurs au moment de choisir leurs députés au Parlement européen. Une enquête préélectorale et une enquête postélectorale confirment que les thèmes relatifs à la situation politique interne d'Espagne ont été déterminants lors du vote dans les mêmes proportions (respectivement $61,3 \%{ }^{13}$ et $59,9 \%{ }^{14}$ ).

\section{Sanction du gouvernement et résistance socialiste}

Validant les prévisions de la majorité des sondages publiés les semaines précédant les élections, les résultats montrent globalement une victoire du $P P(42,1 \%)$ face au $P S O E(38,7 \%)$, inversant de cette manière les résultats obtenus lors des élections européennes de 2004 ou le PSOE dépassait le PP de deux points. La comparaison de ces deux élections doit cependant prendre en compte les contextes économiques et politiques différents des deux élections et conduit à des interprétations différentes des résultats obtenus.

Les élections européennes de 2004 se présentèrent comme des élections de ratification ou de rectification de la victoire inattendue du PSOE lors des élections législatives de mars de la même année. Les élections de 2009 se déroulèrent quant à elles dans un contexte politique plus neutre, moins d'un an après les dernières élections législatives qui confirmèrent au pouvoir le gouvernement socialiste et près de trois ans avant les prochaines élections générales.

Si les élections européennes de 2004 purent être interprétées comme une ratification et un renforcement du nouveau gouvernement présidé par Rodríguez Zapatero, les résultats des élections de 2009 pourraient être considérés comme une sanction modérée à l'égard de ce même gouvernement socialiste. C'est ce qu'indiquent tant les motivations des électeurs des principaux partis (analysées plus haut) que l'écart de suffrages peu important (moins de trois points) entre le PP et le PSOE.

\footnotetext{
12. L'étude $n^{\circ} 2642$ du CIS, réalisée en mai 2006 à l'occasion du $20^{e}$ anniversaire de l'entrée de l'Espagne dans l'Union européenne, montrait que la majorité des citoyens considérait que l'adhésion de l'Espagne avait eu des effets positifs ou très positifs pour la modernisation de la société espagnole $(81,2 \%)$, le fonctionnement de la démocratie $(73,3 \%)$ et le rôle de l'Espagne dans le monde $(74,3 \%)$. La valorisation négative de ces trois items n'atteignait jamais plus de $10 \%$.

13. Voir l'Étude $n^{\circ} 2880$ du CIS (Estudio preelectotal elecciones al Parlamento Europeo) de mai 2009.

14. Enquête postélectorale réalisée par NC Report pour le périodique La Razón (15 juin 2009).
} 
L'évolution dans le temps des résultats des élections européennes montre clairement la tendance au resserrement de la concurrence entre le $P P$ et le $P S O E$ et la relative correspondance entre les cycles des élections nationales et le contexte politique de chaque élection européenne (Graphique 2).

Alors qu'en 1987, 1989, 1999 et 2004, le vote européen apparaissait comme un vote de confiance envers le gouvernement de l'époque (gouvernement socialiste en 1987, 1989 et 2004, et gouvernement du $P P$ en 1999), en 2009 comme en 1994, la victoire du $P P$ peut s'interpréter comme un vote sanction du gouvernement, même s'il faut se garder d'extrapoler le résultat de ces élections européennes à ceux des élections régionales à venir. Il convient en effet de ne pas oublier ce qui fut qualifié de «faux miroir des européennes » lors des élections de 1994 : la large victoire du $P P$ aux élections européennes fut suivie d'une mince victoire - de moins d'un $1 \%-$ aux élections législatives de $1996^{15}$.

Graphique 2 : Pourcentage des votes par partis 1987-2009

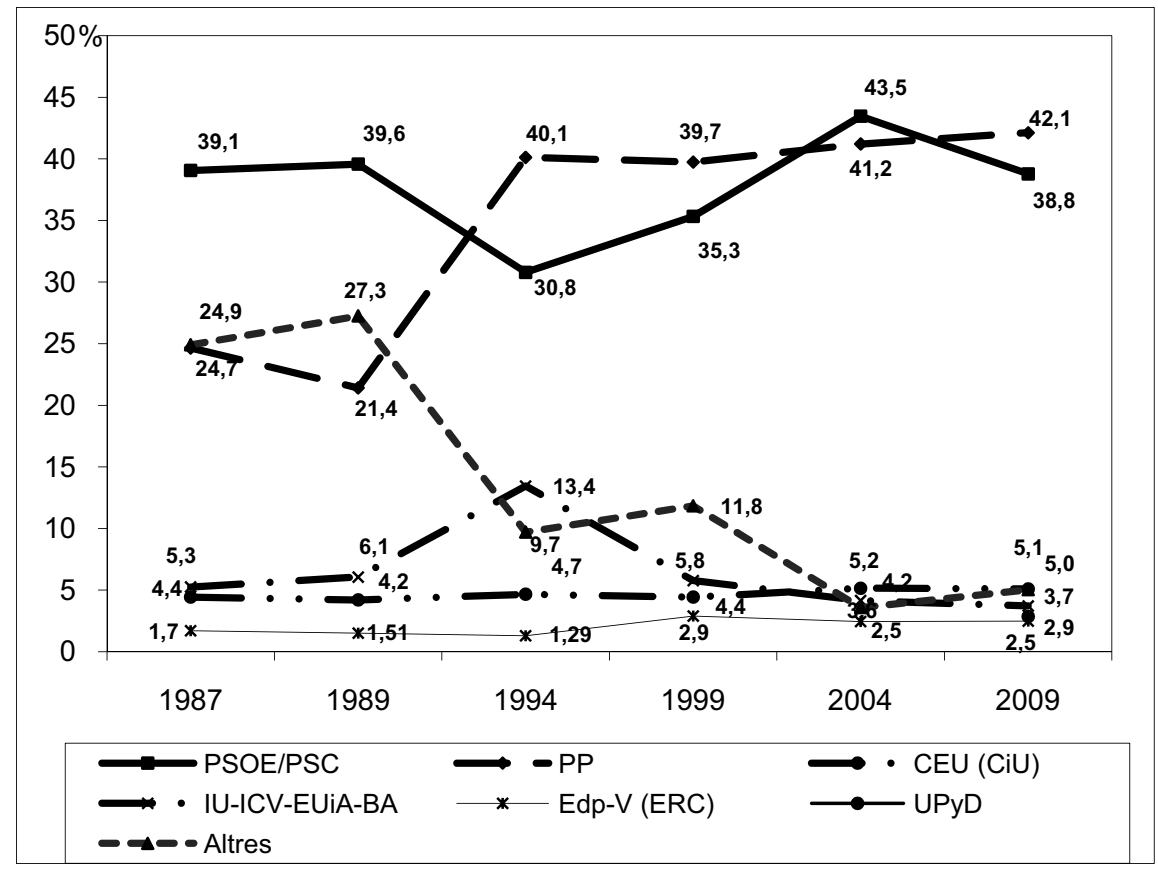

Source : L'auteur à partir des données du ministère de l'Intérieur

Plus généralement et en considérant à la fois le contexte politique, les tendances de vote exprimées dans des situations comparables dans les autres

15. Voir CASTRO C., « Relato electoral de España 1977-2007 », Barcelone (ICPS), 2008, p. 170 et ss. 
pays européens, les prévisions avancées par les enquêtes et sondages préélectoraux, les socialistes espagnols ont-ils «sauvé les meubles »?

L'étude postélectorale publiée par le quotidien El Público une semaine après les élections apportent quelques éléments importants de réponse ${ }^{16}$. Le $P S O E$ a réussi à conserver $59 \%$ des électeurs socialistes de mars 2008 tandis que $32 \%$ de ces électeurs déclarent avoir rejoint les abstentionnistes en 2009. De son côté le PP a conservé $65 \%$ de son électorat de 2008 et en a perdu $28 \%$ qui se sont abstenus. Mais en même temps le $P P$ a réussi à attirer en $200910 \%$ d'électeurs qui s'étaient abstenus ou avaient voté blanc lors des législatives de 2008. Selon toujours ce même étude postélectorale les transferts de voix entre le $P S O E$ et le $P P$ sont infimes (moins d'un $1 \%$ ), tandis que $3 \%$ des électeurs PSOE de 2008 et un $2 \%$ des électeurs $P P$ ont voté pour le nouveau parti $U P y D$. Ces estimations suggèrent aussi une corrélation entre participation et vote au bénéfice du $P P$, ce qu'une analyse territoriale plus détaillée confirme.

En effet, dans les Communautés ou régions qui enregistrèrent une augmentation de la participation par rapport aux européennes de 2004, le vote pour le $P P$ se situe clairement au-dessus de son résultat global pour l'Espagne. C'est le cas dans la plupart des fiefs électoraux de ce parti (Madrid, Castille-Leon, Communauté de Valence et Murcie), et tout particulièrement dans ces deux dernières régions où la progression des votes $P P$ est de $2,6 \%$ par rapport aux résultats de 2004 qui étaient déjà fortement favorables. De plus, le $P P$ améliore légèrement ses scores dans des régions de gouvernement et de majorité socialistes, comme la Castille-Manche et l'Estrémadure (1,7 et $1 \%$ respectivement).

La géographie des votes majoritaires en faveur du PP ou du PSOE (voir cartes 1 et 2) dans chaque Communauté Autonome, montre une distribution des votes identique en 2004 et 2009 et de minces variations par rapport à la carte électorale des législatives du $2008{ }^{17}$. Mais il faut noter le recul asymétrique du vote socialiste dans la Communauté de Valence (- 4,8\%) - d'autant plus notable que le $P P$ progresse dans les mêmes proportions - et également le recul significatif du $P S O E$ en Andalousie, fief principal des socialistes avec la Catalogne.

En Catalogne, le recul de 6,8 points du vote $P S O E$ ne bénéfice pas au $P P$ qui progresse seulement de $0,2 \%$. Le recul socialiste est davantage le résultat de la progression de l'abstention dans l'ensemble de la Communauté

16. Voir « Pobliscopio » dans El Público, 16 juin 2009.

17. Outre la victoire du $P P$ à nouveau dans deux de ces bastions traditionnels, les Iles Baléares et les Iles Canaries, la différence plus importante se produisit au Pays basque, où en 2008 les socialistes gagnèrent dans l'ensemble de la Communauté, alors que lors des élections européennes de 2009, le $P N V$ fut vainqueur dans la Communauté grâce à une légère avance de quelques 7.000 voix sur les socialistes. 
(3,2\% de plus qu'en 2004) et de la progression du parti nationaliste modéré Convergència i Unio $(\mathrm{CiU})$ qui retrouve une grande partie de ses voix perdues en 2004. Il semble s'agir, dans ce dernier cas, d'une faiblesse circonstancielle du vote socialiste catalan qui conserve son pourcentage traditionnel aux élections européennes, proche des $35 \%$.

En Andalousie, le recul socialiste apparaît plus structurel dans la mesure où, par rapport à 2004, ce recul $(-6,2 \%)$ s'accompagne d'un accroissement de plus de trois points du vote $P P$, accentuant le resserrement de la concurrence entre les deux principaux partis déjà observé lors des élections législatives de 2008.

Pour sa part, le $P P$ progresse de manière modérée dans plusieurs Communautés. Outre celles déjà mentionnées qui s'accompagnent de l'augmentation de la participation ${ }^{18}$, le $P P$ croit en Andalousie (+ 3,5\%) et en Galice $(+3,3 \%)$ où il consolide sa victoire des élections régionales d'avril. Malgré son bon résultat général et territorial, le $P P$ perd des voix dans plusieurs Communautés : Asturies, Baléares, Castille-Leon et de manière notable au Pays Basque $(-5,1 \%)$ et en Navarre (- 7,3 \%). Dans ces deux derniers cas, le recul s'explique plus par des facteurs locaux apparus cette dernière année en relation avec la crise interne du $P P$ que par des facteurs politiques liés à l'enjeu électoral européen.

Carte 1 : Parti majoritaire aux élections européennes de 2004 et 2009 par Communauté Autonome

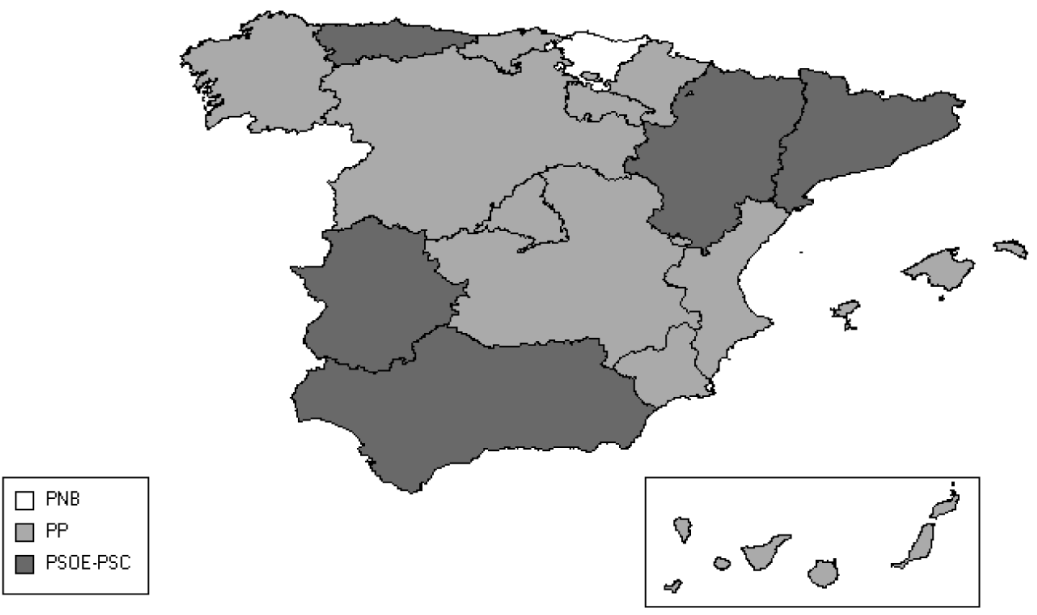

Source : L'auteur à partir des données du Ministère de l'Intérieur

18. Murcie, Communauté valencienne, Castille-Manche, Estrémadure. 
Carte 2 : Parti majoritaire aux élections législatives 2008 par Communauté Autonome

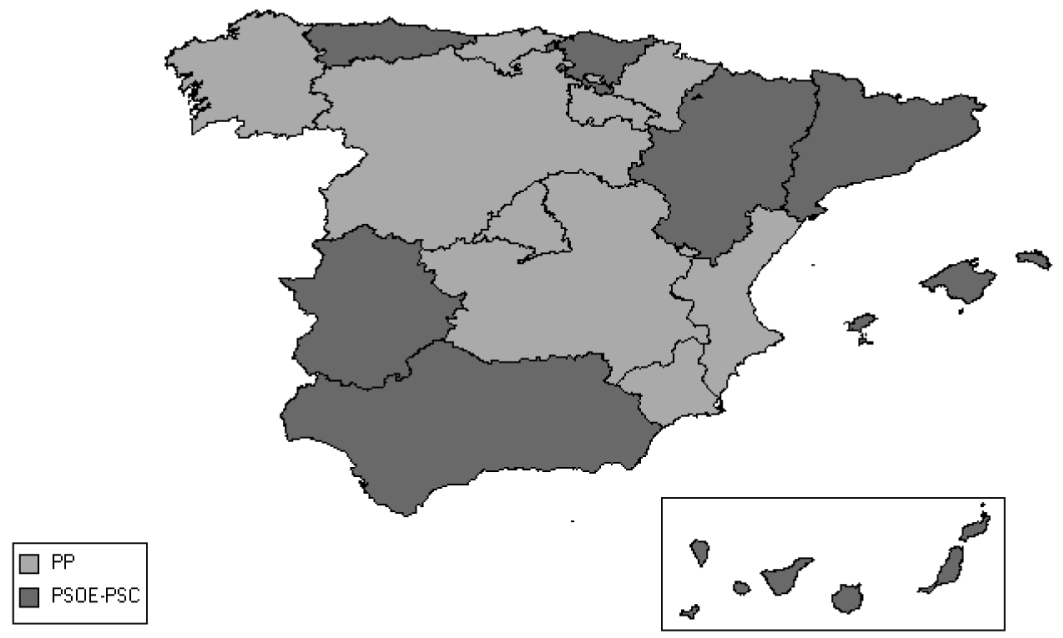

Source : L'auteur à partir des données du Ministère de l'Intérieur

En approfondissant l'analyse territoriale au niveau des capitales provinciales, on constate la bonne tenue du vote urbain en faveur du Partido Popular depuis que celui ci a réussi à conquérir la majorité des grandes villes lors des élections locales du début des années 90 . C'est ainsi que les socialistes ne l'emportent en 2009 que dans seulement 10 des 52 capitales provinciales : les quatre de Catalogne (Barcelone, Girone, LLeida et Tarragone), deux du Pays basque (Vitoria et San Sébastian) - le $P N V$ emportant Bilbao -, deux des trois capitales provinciales de l'Aragon (Saragosse et Huesca) et deux des huit de l'Andalousie (Séville et Huelva). Cette tendance au recul du vote urbain socialiste apparaît le plus clairement dans le cas de la Communauté autonome d'Andalousie, aux élections locales comme dans les élections législatives et celles pour les députés européens.

Le $P P$, vainqueur dans 6 des capitales des provinces andalouses et dans un total de 41 des capitales provinciales de l'ensemble de l'Espagne, semble affermir son implantation urbaine. Cette constatation nuance certainement l'impression de répartition équilibrée des territoires entre $P S O E$ et $P P$ que l'on aurait pu déduire de l'analyse conduite au niveau plus macro territorial des Communautés.

L'analyse territoriale indique en définitive un léger, mais persistant, changement du rapport de forces entre le $P P$ et le $P S O E$ que des études postélectorales de plus grande ampleur et les futures élections devraient pouvoir corroborer. 
En conclusion, nous pouvons souligner que les résultats des élections européennes en Espagne confirment la polarisation PSOE-PP ${ }^{19}$ du rapport des forces politiques. Plus précisément, elles se soldent par un léger recul socialiste par rapport à 2004, territorialement asymétrique et plus important dans les zones urbaines. Elles suggèrent un léger renforcement de la progression du vote $P P$ territorialement plus homogène à l'exception de la Catalogne et du Pays Basque. Enfin pour la première fois s'est exprimé de façon claire un «vote sanction » contre le gouvernement, peu utilisé en Espagne jusqu'à présent mais fréquent dans la majorité des pays de l'Union.

On peut s'interroger pour l'avenir sur la stabilité du gouvernement espagnol compte tenu de la profondeur de la crise économique et des controverses qui accompagnent la gestion gouvernementale. Mais, comme nous l'avons souligné, l'expérience des mirages déjoués lors des précédents rendez vous électoraux conseille une bonne dose de prudence et incite à une profondeur accrue dans l'analyse électorale pour scruter le futur.

19. Le total des votes pour le $P S O E$ et le $P P$ atteignait $92 \%$ lors des élections de 2008 et dépasse les $80 \%$ lors des élections européennes de 2009 , ce qui confirme une tendance stable constatée ces dernières 20 années. 